\title{
Towards a System Support of Collaborative Knowledge Work
}

\author{
Nicolas Mundbrod, Jens Kolb and Manfred Reichert \\ Institute of Databases and Information Systems \\ Ulm University, Germany \\ \{nicolas.mundbrod, jens.kolb, manfred.reichert\}@uni-ulm.de \\ http://www.uni-ulm.de/dbis
}

\begin{abstract}
Knowledge work is becoming the predominant type of work in developed countries. Leveraging their expertise, skills, and experiences, knowledge workers daily deal with demanding situations. Therefore, they widely work autonomously, but usually collaborate in multiple contexts. Further, their work is influenced by dynamic factors like time constraints, costs, and available resources, and thereby it cannot be pre-specified like routine work. The lack of an appropriate context and process support, in turn, reduces their productivity and hinders the reuse as well as the continuous improvement of elaborated solutions. This paper structures collaborative knowledge work and presents its characteristics and dimensions. Moreover, we introduce a lifecycle methodology to support collaborative knowledge workers holistically.
\end{abstract}

Key words: Knowledge Work, Knowledge Workers, Collaboration, Lifecycle Support, CSCW

\section{Introduction}

A change from an industrial towards a knowledge-based society is taking place in developed countries: knowledge work is becoming the predominant type of work 1. Utilizing their distinguished skills, gained experiences, and expertise, knowledge workers (e.g., doctors, engineers, or researchers) daily solve demanding and sophisticated tasks. Hence, knowledge work represents an important part of today's corporate business processes. Contemporary process-aware information systems (PAISs) are not able to support knowledge workers adequately as pre-specified business process models are required. These models cannot be provided since knowledge work's tasks and their order are not predictable in detail. Instead, knowledge workers have to manually interrelate process-related information encapsulated in a variety of heterogeneous software systems (e.g., social and special-purpose software). Hence, there is no knowledge work assistance comparable to the support a PAIS can provide for routine work.

Based on 2, this paper discussed how collaborative knowledge work (CKW) can be supported by an adaptive information system (CKW system) (objective $I)$. For this purpose, a sound definitional framework of CKW is established and 
CKW characteristics and dimensions are presented based on a case study (objective II). The remainder of this paper is structured as follows: Section 2 introduces the fundamentals of CKW. Subsequently, Section 3 presents representative CKW use cases. Section 4 provides CKW characteristics and dimensions. Section 5 introduces a lifecycle methodology for CKW. Finally, Section 6 summarizes the results and gives insights into future research.

\section{Fundamentals of Collaborative Knowledge Work}

\subsection{Knowledge}

Generally, the term of knowledge does not obtain a unique definition [3. However, from the perspective of computer science, it is desirable to classify the terms of data, information and knowledge as those are often used synonymously by mistake. Data represents syntactic entities comprising a set of symbols (with no meaning), whereas information represents the output from data interpretation and hence data with meaning (i.e., semantics) [4. Finally, knowledge is learned information, incorporated in an agent's reasoning resources (cf. Definition 1).

Definition 1. Knowledge is a fluid mix of framed experiences, values, contextual information, and expert insights that provides a framework for evaluating and incorporating new experiences and information. It originates and is applied in the minds of knowers. In organizations, it often becomes embedded, not only in documents or repositories, but also in organizational routines, processes, practices, and norms. [5]

For every collaboration, it is crucial that knowledge is communicable, enabling knowledge workers to easily share it. Already implied in Definition 1. epistemological scientist Polanyi shaped a distinction between tacit and explicit knowledge through the phrase "We can know more than we can tell" 6]. One can hold tacit knowledge without having the capability to explicitly express the quintessence, e.g., the capability to hold the balance on a bicycle. In contrast, explicit knowledge is expressible in a formal, systematic language and therefore it can be regarded as communicable knowledge (i.e., information). The differentiation provides the basis for Nonaka's and Takeuchi's theory of (corporate) knowledge creation 7 . Four different conversion modes between tacit and explicit knowledge are described: socialization (i.e., from tacit to tacit knowledge), externalization (i.e., tacit to explicit), combination (i.e., explicit), and internalization (i.e., explicit to tacit). A constant repetition of the different conversions provides the basis of the individual and organizational knowledge generation process: knowledge is spirally advanced and transferred from being individually obtained to organizationally or even inter-organizationally shared (cf. Figure 1). 


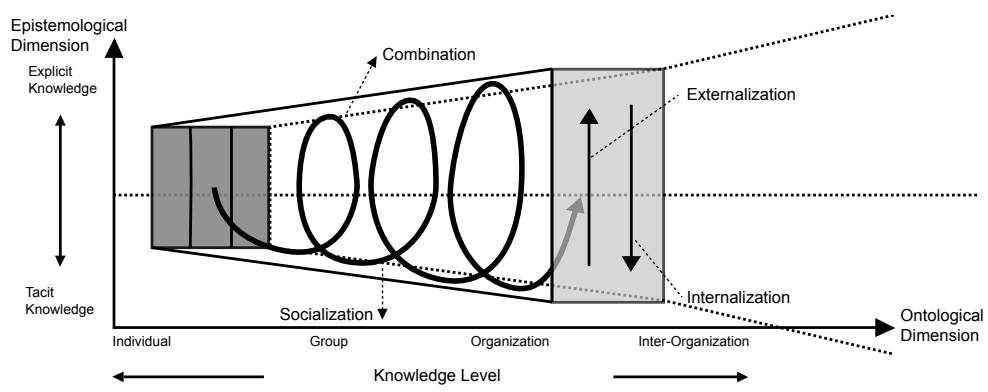

Fig. 1: Organizational Knowledge Creation Spiral [7]

\subsection{Knowledge Work}

Hube assessed 16 definitions of knowledge work in relation to four self-established criteria: applicability, process description, context relation, and distinction [8]. He suggests Definition 2 as the most appropriate one for knowledge work ${ }^{1}$ [ 8 .

Definition 2. Knowledge work is comprised of objectifying intellectual activities, addressing novel and complex processes and (work) results, which require external means of control and a dual field of action.

Hube separates the term of knowledge work from the related term of intellectual work. While intellectual work generally contains mental work, knowledge work comprises only activities addressing novel and complex processes as well as work results. Hence, routine mental work is excluded. Based on the theory of action regulation, an individual performing knowledge work is supposed to use a referential and an actual field of action [9]. In the referential field of action, one merely deals with a problem theoretically. Thereby, one can deliberately act and test different approaches, not causing any impact on the assignment. In comparison, necessary instruments, actions and resources are used in the actual field of action to properly manage the complex processes. Further, the results of the referential field of action are transferred to finally achieve the work's objective.

\subsection{Knowledge Work Process}

Due to knowledge work's focus on novelty and complexity, obviously, there is no pre-specified process an individual can perform. However, a generic and idealtypical knowledge work process is described as shown in Figure 2. Note that each of the process steps can be executed multiple times and might be skipped as well. First, an individual performing knowledge work deducts an assignment from an ambition and available information (Step 1). Subsequently, orientation (2) and planning (3) in the actual field of action take place, the assignment is

\footnotetext{
${ }^{1}$ Definition 2 is translated from German.
} 
concretized, and possible resources are evaluated. Then, theoretical solutions are reviewed (4) and a solution strategy is designed (5). Consequently, the plan is put into operation (6), and the intermediate work result is constantly assessed. On the one hand the result is evaluated considering its formal quality (7), on the other hand the original plan might be adapted (8). Additionally, it is even possible that the whole assignment has to be re-evaluated (9).

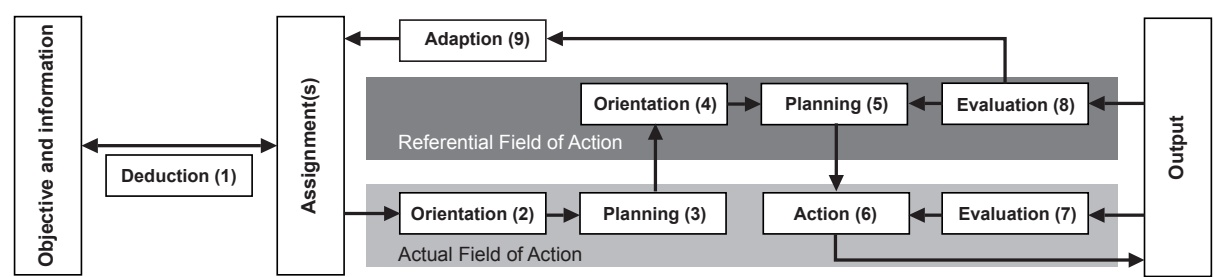

Fig. 2: Generic Knowledge Work Process [8]

\subsection{Knowledge Workers}

Naturally, a knowledge work process highly depends on the knowledge of the individuals who perform knowledge work. There are various definitions addressing the question who exactly is a knowledge worker or not (cf. 8, 10, 11]). Concluding, Definition 3 introduces the term of a knowledge worker [12].

Definition 3. Knowledge workers have high degrees of expertise, education, or experience, and the primary purpose of their jobs involves the process and accomplishment of knowledge work.

Obviously, whether an individual rates a task to be routine or rather novel and challenging, depends on his personal degree of foreknowledge. Knowledge workers exposing high degrees of expertise, education, and experience usually deal better with novel and complex situations as others without respective foreknowledge. This coherence rounds out the connection between Definitions 2 and 3 . Generally, knowledge workers comprise various professions: A manager of literally any company is supposed to perform knowledge work in order to successfully manage and improve a company's business [12]. Further, Definition 3 underlines that knowledge workers hold responsible positions, i.e., their productivity is a crucial concern for any company.

Usually, the processing of novel and complex problems is split into manageable parts, which are assigned to knowledge workers who feature the needed expertise and experiences [8]. However, based on their expertise and experiences, knowledge workers are concurrently requested in multiple contexts, where they need to adopt different roles. This exposes the main issues knowledge workers face these days: they have to manually filter, classify and manage context-related 
information to project their thoughts into the corresponding context. Furthermore, they must cope with the related issue of attention fragmentation, while trying to keep track of any progress being made in different context. In order to adequately inspect the properties of CKW, Definition 4 defines it formally.

Definition 4. Collaborative knowledge work (CKW) is described as knowledge work jointly performed by two or more knowledge workers in order to achieve a common business goal.

\section{Collaborative Knowledge Work Use Cases}

To validate the definitions, three different CKW use cases are introduced ${ }^{2}$ Based on these, CKW characteristics and dimensions are derived in Section 4

UC1: Development Project. This use case is based on a development project for an embedded system in the automotive sector. It features multidisciplinary collaboration of knowledge workers, e.g., vehicles require complex mechatronic systems developed by engineers of various professions. Typically, a best practice (e.g., a V-model) is used to systematically synchronize results of concurrent development teams (i.e., qualitative development course) and to provide an understandable overview whereby knowledge workers can orientate. Moreover, a virtual system prototype is created and gradually enhanced. Development, research, and implementation projects can be generally considered to deal with the well-organized creation of solutions (i.e., explicit knowledge) considering a pre-defined problem. Complex problems are extensively studied, analyzed, and evaluated up-front to collaboratively develop a solution based on a deployed project methodology (e.g., a domain-related best practice).

UC2: Criminal Investigations. Criminal investigations are addressed in this use case: "an investigation is the examination, study, tracking, and gathering of factual information that answers questions or solves problems" [13. Investigative work often contains several concurrently emerging angles with dedicated investigative staff members ascertaining. While there are standardized investigative actions (e.g., securing of evidence), investigators in charge have to individually determine which of the standard procedures need to be applied. Comparably, the work of attorneys, judges and researchers is naturally connected to the work of public investigation authorities. Moreover, companies are increasingly obliged to provide information on requests of customers, citizens, regulators, or board members (e.g., audit requests or fraud detection).

UC3: Complex Financial Service Request. Business processes in the financial service sector are highly standardized today. However, there are still exceptional situations which have to be handled individually. Presumably, a financial service company receives a request for a large-scale combination of

\footnotetext{
${ }^{2}$ Detailed descriptions of the use cases are available in [2].
} 
financial products from a key customer. Therefore, financial experts, who are specialized to specific products, are involved on demand by the responsible key account manager to meet the customer's needs. In this case, related use cases are insurance claim handling, product change requests, loan origination, customer onboarding, and intensive patient care. All these have in common that they are dependent on human assessment and decisions based on expertise and experiences, continuously gained information and the proper handling of unexpected events and occurring problems.

\section{Characteristics and Dimensions}

Based on the use cases (cf. Section 3), characteristics (cf. Section 4.1) and dimensions (cf. Section 4.2 are presented. Figure 3 gives an overview of the CKW characteristics and their coherence.

\subsection{CKW Characteristics}

C1: Uncertainty. The notion of complexity, implicitly addressed by knowledge work, refers to problems or situations comprising an unmanageable set of influencing factors intertwined via dynamic correlations [8]. Consequently, the generic knowledge work process (cf. Section 2.3p includes three feedback loops that are motivated by the need to continuously assess the planned and finally conducted actions. The course of actions is dynamically determined by the involved knowledge workers and based on their expertise and experiences. Considering the collaboration of knowledge workers, obviously, labor division comprises interdependencies and mutual interference between involved knowledge workers which further increase the general dynamics.

C2: Goal Orientation. A common goal, e.g., to meet customer's needs (cf. UC3), can be considered as the integrative factor for knowledge workers. In relation, Drucker stated that the crucial question in a knowledge worker's productivity is "what is the task?" 14. In UC2, investigators collaborate to solve a crime and thereby derive the required tasks. Ideally, knowledge workers' individual goals are well integrated into the scope of a common goal. To adequately cope with complex and unpredictable CKW, sub-goals 3 are derived that are achievable in a shorter period of time. While a common goal in CKW should remain rather stable, sub-goals can be modified or even removed.

C3: Emergence. While pursuing a common goal and addressing uncertainty, knowledge workers continually adapt activities to successfully achieve their subgoals. As a result of $\mathrm{C} 1$, knowledge workers have to focus on the planning of activities conducted any time soon (proximity of time). Activities scheduled later on might be brought up in principle, but not defined in detail yet. This

\footnotetext{
${ }^{3}$ Also commonly known as milestones.
} 


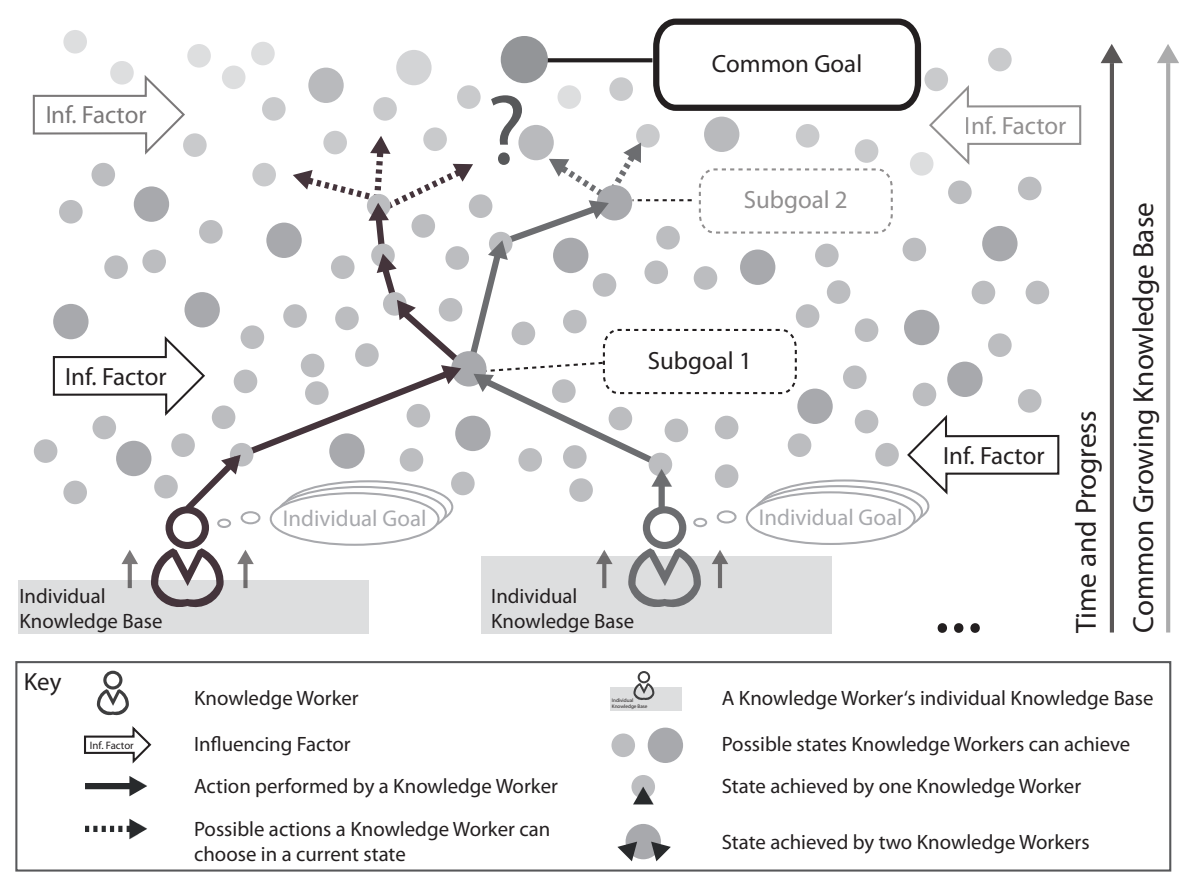

Fig. 3: CKW Characteristics and their Coherence

agile planning and working implicates that CKW processes gradually emerge: knowledge workers constantly evaluate possible activities on the basis of their current state and in consideration of influencing factors. At every point, they have the choice between several performable actions to achieve further states and to further approach their common goal (cf. Figure 3).

C4: Growing Knowledge Base. Externalization of knowledge in the shape of communicable information is crucially needed to achieve organizationally shared knowledge - a prerequisite for collaboration (cf. Section 2.1). CKW's explicit knowledge base usually comprises heterogeneous information, which has to be managed properly (e.g., office documents, e-mails, and handwritten notes). In general, the progress of a use case towards its goal is strongly connected to the advancement of the tacit and explicit knowledge base. For instance, information like schedules, responsibilities and methodologies is stepwise created to organize the project at the beginning of UC1. Afterwards, a prototype or developed components can represent the current state of development.

\subsection{Dimensions}

Naturally, countless dimensions can be considered by which CKW scenarios could be differentiated. Hence, this section focuses on dimensions clearly exposing significant implications for the system support of CKW. 
D1: Knowledge Action Type. CKW can be differentiated by the predominant way the knowledge workers deal with knowledge and information. In relation, Davenport distinguishes between the knowledge actions acquisition, application, creation, dissemination, documentation, and packaging of knowledge [15. Further types are presented in [16, 17]. Although there are different approaches, pragmatic analyses of the main knowledge actions can yield benefits as the support of CKW can be adjusted accordingly.

D2: Methodology. The degree of adherence to a common methodology discernibly varies in UC1-UC3. The automotive development team (UC1), for example, decides to apply a V-model to organize CKW and to ensure a qualitative development progress. While individual activities are still subject to the different knowledge workers, an overall defined methodology is given. Methodologies do not have to be explicitly illustrated, renowned or even described to be successfully applied: a team can follow a tacit methodology, known and accepted by all knowledge workers as well as robust in the face of varying conditions.

D3: Interdisciplinarity. The use cases UC1-UC3 additionally unveiled that CKW varies from domain-specific to interdisciplinary work. CKW involving different domains can lead to misunderstandings, discords (e.g., about common procedures), or even severe inconsistencies. In this context, synonyms and homonyms can result in high effort to synchronize contributions. However, especially interdisciplinary work is highly promising for novel and complex issues.

D4: Organizational Frame. CKW is not compulsorily bound to organizational units or hierarchical structures. It can be distinguished by the surrounding organizational frame as well. In general, knowledge workers usually collaborate either spontaneously (i.e., without a dedicated organizational frame), or based on organizational frames like a case or project. Especially coordination aspects (i.e., responsibilities, organizational models, work allocations, and synchronizations) are influenced by the surrounding organizational frame.

D5: Spatial Proximity. Apart from preferred knowledge actions and organizational frames, CKW depends on the fact whether knowledge workers can directly communicate with each other, or not. Physical closeness empowers knowledge workers to directly communicate face to face, whereas physically separated knowledge workers obviously have to rely on communication and collaboration tools to virtually bridge a spatial gap. Hence, CKW can be distinguished by the degree of spatial proximity the knowledge workers have during their collaboration.

D6: Involved Knowledge Workers. The number of involved knowledge workers can vary between CKW projects as well. The complexity of CKW is a driver of the knowledge workers' headcount. Moreover, the corporate importance of CKW may be another reason to include more knowledge workers. In general, the number of involved knowledge workers results in an increased demand for appropriate support, especially for the systematic allocation and synchronization of work (coordination). 
D7: Temporal Constraints. Time constraints [18 may also distinguish CKW projects. For example, fixed deadlines can be initially connected with objectives (i.e., fixed time frames). Considering $\mathrm{UC} 2$, there are sometime only relative or even no fixed deadlines, but investigators still suffer from a time pressure to solve the crime. In general, some CKW projects may be scheduled for months or even years whereas others have to be finished within hours or days.

D8: Information Interdependency. The acquisition of information to detect causal relationships can be regarded as the main purpose of investigative activities (cf. use case UC2). Closely related to dimension D1 and characteristic C4, CKW can also be distinguished by the complexity and importance of information interdependencies. Apart from internal information interdependencies in a company, CKW projects can also feature coordinative and information interdependencies between each other. Furthermore, the degree of interdependencies also raises coordination efforts.

D9: Number of Repetitions. The number of repetitive occurrences provides another dimension to distinguish CKW. As CKW is characterized to be emergent and rather unique, the dimension might sound curious. However, when common goals are considered in detail, a repetitive occurrence of CKW can be observed. Apart from a common goal, the presented dimensions D1-D8 can also be utilized to determine whether CKW projects share common properties. In general, the provision of a specific support for CKW depends on the possibility to determine the level of similarity an ongoing collaboration shares with already finished CKW. Thereby, it has to be assessed which parts of past scenarios can be leveraged for the support of the ongoing collaboration (i.e., sustainable support).

\section{Collaborative Knowledge Work Lifecycle}

In order to systematically support CKW by a dedicated information system ( $C K W$ system), the availability, advancement and communication of knowledge have to be ensured. If knowledge workers are empowered to quickly retrieve context-relevant information as well as experiential knowledge in the right shape and at the right point of time, their efficiency and effectiveness can be increased 8]. To establish a context- and process-aware support, the BPM lifecycle 19] can be leveraged: the Collaborative Knowledge Work Lifecycle (CKWL) (cf. Figure (4) describes integral phases, a CKW system has to provide.

The lifecycle consistently draws on the generic knowledge work process (cf. Section 2.3 as it features the phases Orientation ${ }^{4}$. Template Design, Collaboration Run Time, and Records Evaluation. Further, it includes action regulation (cf. Section 2.2 implemented by a feedback loop (i.e., knowledge retrieval). The subsequent sections discuss the different lifecycle phases in detail.

\footnotetext{
${ }^{4}$ The CKWL is entered in the orientation phase
} 


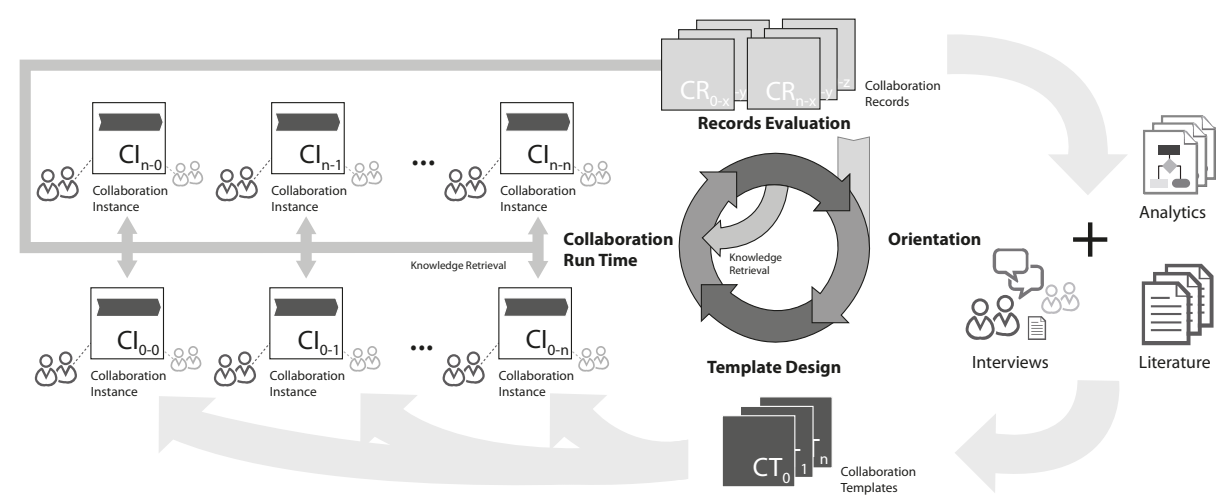

Fig. 4: The Collaborative Knowledge Work Lifecycle

Orientation Phase. Information about how knowledge workers usually collaborate in a certain context has to be gathered in the orientation phase. Dimension D9 implies that CKW can be regarded to occur multiple times and hence be aggregated to a dedicated collaboration type. To perform a sound aggregation, dimensions D1-D8 can be utilized. Moreover, records of finalized collaborations are leveraged, involved knowledge workers systematically interviewed, or subjectrelated literature and expert experiences taken into consideration. In general, while the flow of activities is the main subject in the design phase of the BPM lifecycle, the orientation phase focuses on the knowledge workers' information flow. So, data sources and relevant information systems need to be explicitly documented and integrated [20. Besides identifying information sources, the main knowledge actions of knowledge workers have to be considered as well (cf. D1). Further, their communication structure has to be addressed, especially in case that knowledge workers are distributed and need to communicate remotely (cf. D5). Coordination aspects also have to be taken into account: for instance, commonly used methodologies, organizational frames and frequently arising tasks must be documented. Finally, awareness information knowledge workers require to initiate, perform and manage communication and coordination has to be covered, as well.

Template Design Phase. Based on a thorough examination of collaboration types and their implicit information flows, collaboration templates $(C T)$ need to be created. Thereby, a CT is comparable to a process model supporting standardizable work. A certain $\mathrm{CT}$ is then leveraged as a blueprint for a range of collaboration instances (CIs). A CI refers to a virtual unit supporting CKW, e.g., a specific development project. The involved knowledge workers are initially supported by providing access to the content they likely require when performing their work. However, in comparison to a process model, a CT neither prescribes a set of activities nor their ordering. Predominantly, it is supposed to provide information access, communication, and coordination support embedded in an 
adaptable and growing CKW system. Further, a CT features a goal for the optimal collaboration between knowledge workers in relation to their current context. Logically, a CT has to be highly adaptable and carefully designed in order to support knowledge workers without obtruding or even restraining them.

Collaboration Run Time Phase. Knowledge workers can instantiate a CT according to their preferences and within their current CKW context. If there is no adequate $\mathrm{CT}$ available, knowledge workers can choose a rather generic CT. Moreover, CTs have to be highly adaptive to empower knowledge workers to conduct a wide range of changes without being overstrained by technical details and issues. Knowledge workers are supposed to fully utilize the defined CT to collaborate towards the achievement of their common goal in CKW. On the basis of available information, knowledge workers can communicate and coordinate using communication and coordination features of a supportive system, or additionally available, context-related integrated systems. Finally, the effectiveness and efficiency of knowledge workers' collaboration depends on the provision of experiential knowledge as well. Knowledge workers need to be able to access collaboration records ( $C R$; i.e., finalized CIs) in order to retrieve important information, i.e., knowledge that can substantially facilitate and speed up the achievement of a common goal.

Records Evaluation Phase. CRs can be considered as an important common knowledge base for CIs and knowledge workers involved. Knowledge workers can look up details about past CKW and benefit from documentations. Furthermore, information and their interdependencies from a particular CI can be compared with information and connections available in the archived CRs. In addition, CRs can be used for advancing existing CTs as well as for developing new ones. Moreover, a finished and archived CI can also be used as starting point for a succeeding CI, drawing upon the achieved results and established knowledge base. Naturally, a CKW system should check which parts of a specific CT have been adapted during run time or not been used. Moreover, involved knowledge workers may be interviewed to rate the importance and relevance of information for future endeavors.

\section{Conclusion}

This paper provides sound definitions in terms of knowledge work, knowledge worker and $C K W$ for future use in the scope of BPM (objective II). The definitions are validated by assessing representative use cases, resulting in CKW characteristics and dimensions that can be leveraged to underpin future CKW support. For the latter, a generic CKW methodology is introduced by the CKWL (objective I) aiming at significant improvement of knowledge workers' productivity.

As a conclusion, the holistic and process-oriented support for collaborative knowledge workers is a challenge in the literal sense. Although there is a broad 
range of available technologies targeting single aspects, the integration of those into a utilizable support implies high efforts and distinguished concepts for the interplay of these technologies. However, the provided aspects may be used as a vision to gradually extend and interconnect concepts and technologies towards an intended holistic support according to the CKWL.

\section{References}

1. Pfiffner, M., Stadelmann, P.: Wissen wirksam machen. Haupt Verlag, Bern (1998)

2. Mundbrod, N.: Business Process Support for Collaborative Knowledge Workers. Master thesis, Ulm University (2012)

3. Alavi, M., Leidner, D.E.: Review: Knowledge Management and Knowledge Management Systems: Conceptual Foundations and Research Issues. MIS Quarterly 25(1) (2001) 107-136

4. Aamodt, A., Nygard, M.: Different Roles and Mutual Dependencies of Data, Information, and Knowledge - An AI Perspective on their Integration. Data and Knowledge Engineering 16(3) (1995) 191-222

5. Davenport, T.H., Prusak, L.: Working Knowledge. Harvard Business School Press, Boston (2000)

6. Polanyi, M.: Tacit Dimension. Peter Smith Publisher Inc, Gloucester, Mass. (1983)

7. Nonaka, I., Takeuchi, H.: The Knowledge-Creating Company. Oxford University Press, New York (1995)

8. Hube, G.: Beitrag zur Beschreibung und Analyse von Wissensarbeit. PhD thesis, University of Stuttgart, Stuttgart (2005)

9. Resch, M.: Die Handlungsregulation geistiger Arbeit. Huber, Bern (1988)

10. Tiemann, M.: Wissensintensive Berufe. Bundesinstitut für Berufsbildung, Bonn (2010)

11. Swenson, K.D.: The Nature of Knowledge Work. In Swenson, K.D., ed.: Mastering the Unpredictable. How Adaptive Case Management Will Revolutionize the Way That Knowledge Workers Get Things Done. Meghan-Kiffer-Press (2010) 5-28

12. Davenport, T.H.: Thinking for a Living: How to Get Better Performance and Results from Knowledge Workers. Harvard Business School Press (2005)

13. Sennewald, C.A., Tsukayama, J.K.: The Process of Investigation. ButterworthHeinemann ET - 3 TS - WorldCat, Burlington, MA (2006)

14. Drucker, P.F.: Management Challenges for the 21st Century. Elsevier Science \& Technology, Oxford (2007)

15. Davenport, T.O.: Human Capital. Jossey-Bass, San Francisco (1999)

16. Holsapple, C.W., Jones, K.: Exploring Primary Activities of the Knowledge Chain. Knowledge and Process Management 11(3) (2004) 155-174

17. Reinhardt, W., Schmidt, B., Sloep, P., Drachsler, H.: Knowledge Worker Roles and Actions-Results of Two Empirical Studies. Knowledge and Process Management 18(3) (2011) 150-174

18. Lanz, A., Weber, B., Reichert, M.: Workflow Time Patterns for Process-aware Information Systems. In: BPMDS 2010 and EMMSAD 2010, Hammamet, Tunisia, Springer Heidelberg (June 2010) 94-107

19. Reichert, M., Weber, B.: Enabling Flexibility in Process-Aware Information Systems: Challenges, Methods, Technologies. Springer, Berlin Heidelberg (2012)

20. Michelberger, B., Mutschler, B., Reichert, M.: A Context Framework for Processoriented Information Logistics. In: 15th Int'l Conference on Business Information Systems (BIS 2012), Vilnius, Lithuania, Springer Heidelberg (May 2012) 201-212 